\title{
New FESEM Design for 1nm at 1kV Imaging, EDS and BSE Nanoanalysis, and a Discussion of Diffraction Limits, Depth of Field and the Future
}

\author{
E D Boyes \\ DuPont Company, CR\&D, PO Box 80356-383, Wilmington, DE 19880-0356, USA
}

A novel approach to FESEM design integrates new levels $(>2 \mathrm{x})$ of low voltage image resolution $(\sim 1 \mathrm{~nm}$ at $1 \mathrm{kV}$ and $<0.5 \mathrm{~nm}$ at $20-30 \mathrm{kV})$ with greatly improved $\left(15 \mathrm{x} / 0.3 \mathrm{sr} / 30 \mathrm{~mm}^{2}\right)$ sensitivity for EDS elemental nanoanalysis and chemical imaging, with spatial resolutions down to <100nm and in favorable cases with sensitivity limits of 1-10nm, while retaining the many advantages of robust and representative wide area (mms) bulk specimens. High resolution and sensitivity backscattered $(\mathrm{Z})$ imaging and BSE nanoanalysis are being added. The goal of this project is to extend the practical capabilities of the FESEM as far as possible with component technologies available at the end of the 20th century, and with our current understanding of the laws of physics as they apply to SEM.

The heart of the novel DuPont/Hitachi S5000SPX or X-scope instrument combines a cold field emission gun (FEG) aggressively processed for high emission stability $(<1 \%$ per hour variation in probe current can be achieved) and a new design of final lens with record breaking (in any electron optics ?) low levels of aberrations down to Cs $\sim 0.1 \mathrm{~mm}$ and $\mathrm{Cc}<0.2 \mathrm{~mm}$ (Fig.1). These low values are achieved by natural means; i.e. without correctors. The position of the specimen in the lens is changed to optimize selected experimental parameters. Figs. 1 and 2 illustrate the parameter space. The highest tilt range (currently $+/-25^{\circ}$ ) is achieved at the gap center with $\mathrm{Cs}=0.8 \mathrm{~mm}$ and a resolution of $\sim 1.5 \mathrm{~nm}$ at $1 \mathrm{kV}$. The corresponding collection angle with the special $30 \mathrm{~mm}^{2}$ Noran ATW EDS detector is $0.3 \mathrm{sr}$; and about $15 \mathrm{x}$ that of a typical conventional SEM. To optimize low voltage $(<5 \mathrm{kV})$ imaging with limited tilt and restricted EDS the sample is positioned $<0.5 \mathrm{~mm}$ from the face of the upper polepiece with Cs $\sim 0.1 \mathrm{~mm}$ and a secondary electron image resolution $\sim 1 \mathrm{~nm}$. The very low aberrations are achieved by focusing the probe with only a small fraction of the highly excited lens field.

In principle much better natural aberrations $(\mathrm{Cs}, \mathrm{Cc})$ can be achieved at low voltages in SEM than at high ones in TEM. Absolute excitations (AT=4200) and the accompanying thermal and magnetic saturation issues remain reasonable with relative excitations $\left(\mathrm{NI} / \mathrm{V}_{\mathrm{R}}^{1 / 2}>100\right)$ which would be astronomical for a $200 \mathrm{kV}$ STEM. In the third and fourth zone modes, which may be practical only at low voltages, natural aberrations can be achieved which are superior to anything possible without correctors at $100-200 \mathrm{kV}$. The final limit in LVSEM is access for the sample, stage and detectors. The SEM application is greatly simplified in that the electron beam forming the probe enters on the same side of the lens field as the electron imaging signals exit towards the (in-lens) detectors in the upper column. With a bulk specimen only about $10 \%$ of the total lens field is used to focus the probe and what would have happened if the electron beam had continued further into or through the specimen is of no concern. In comparison to a $1 \Delta$ STEM at $200 \mathrm{kV}$, the $1 \mathrm{~nm}$ LVSEM with a cold field emitter is in principle even more sensitive to AC magnetic fields. Other engineering and environmental factors are also important. Another significant limitation on very short focal length and high resolution operations is the need to maximize the aperture size to avoid diffraction limits intruding, especially at low and very low voltages. The serious trade off is in depth of field. This remains workable at $\sim 20 \mathrm{~nm}$ at $1 \mathrm{kV}$ (and $\sim 1 \mathrm{~nm}$ resolution at 300,000x, Fig.3) but it obviously is no 
longer as extensive as in classical SEM operations at more modest resolution. This would seem to set a practical limit on what we could hope to achieve even with an aberration corrected system; although the greater flexibility in specimen access and WD this would make possible, with some other complications, at the current resolution would have value, especially for in-situ experiments.

The TEM-style jeweled rod specimen holders for the X-scope are innovative, defy conventional wisdom at no more than $1 \mathrm{~mm}$ thick, are mostly home built, and with some operational restrictions are reverse compatible with older S5000 systems. Samples are preferably $<0.5 \mathrm{~mm}$ thick and $2 \mathrm{~mm}$ is the maximum. The end sections of the holders are electrically isolated with provision for probe current measurements and continuous touch alarms and stage motor interrupts ensure safe tilting to optimum angles. The regular holders for $3 \mathrm{~mm}$ disks or grids allow $+/-25^{\circ}$ of tilt as do the ones for $4.5 \times 8.5 \mathrm{~mm}$ plates, and a stage limited $60^{\circ}$ of tilt is available with $2.3 \mathrm{~mm}$ wide plates and disks. For operation at the highest $\mathrm{z}$ positions with very low Cs $(\sim 0.1 \mathrm{~mm})$ and very short WD $(<0.5 \mathrm{~mm})$ special holders offset the specimen position above the eucentric tilt axis of the stage.

The design of (Fig.2) of the optics, vacuum system, specimen chamber, stage and specimen holders, and the SEI, BSE and ATW EDS detectors, have been fully integrated in the ab initio design of the new system to great benefit and minimum compromises; except explicitly in specimen size which so far is not proving to be very restrictive in applications with particles, pigments, fibers, catalysts.

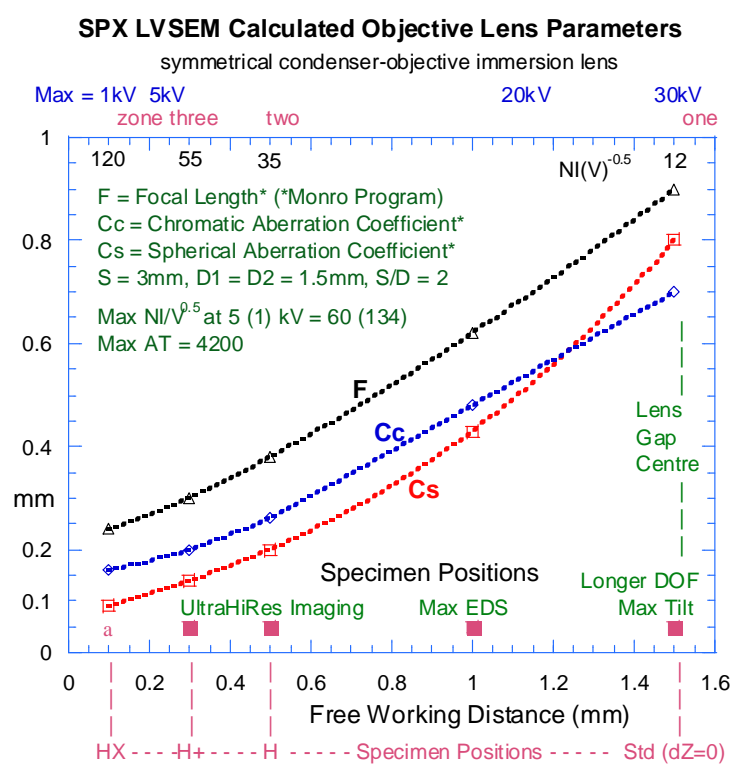

Fig.1 : Cs, Cc, F calculated for new X-scope

Fig.2: X-scope specimen area schematic $\rightarrow$

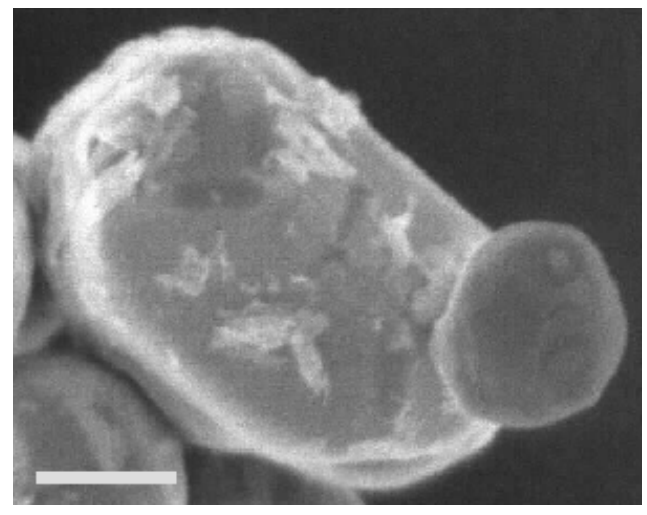

Fig. 3 : Partially coated ceramic particle at $1 \mathrm{kV}$ Scale is $60 \mathrm{~nm}$, original mag $150,000 \mathrm{x}$

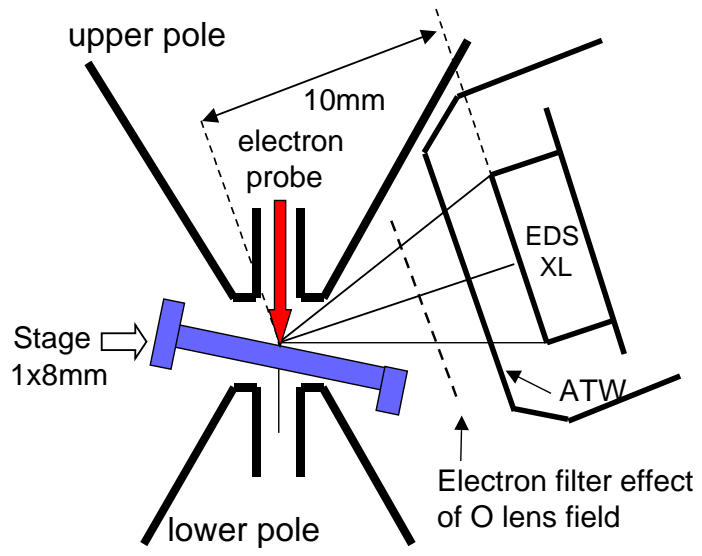

